\title{
RECENSIÓN
}

\section{REVISIONES EN SALUD PÚBLICA}

\author{
M. Porta Serra, C. Álvarez-Dardet y E. Fernández Muñoz \\ Editores.
}

Año 1997

Masson. Barcelona

ISBN 84-458-0554-1

248 páginas.

Recensión: Cristina Pérez. Andrés. Subdirección General de Epidemiología. Promoción y Educación para la Salud. Dirección General de Salud Pública. Ministerio de Sanidad y Consumo.

Se ha publicado el quinto volumen de la colección que bajo el título Revisiones en Salud Pública, se edita con una frecuencia bianual, desde el año 1989.

En la presentación de este último número, la cual está firmada por sus tres editores, $\mathrm{M}$. Porta Serra, C. Álvarez-Dardet y E. Fernández Muñoz, manifiestan éstos su interés por el género del ensayo frente a la revisión anglosajona clásica y del metaanálisis, a los que, sin embargo, no desmerecen. En relación con este interés por el ensayo aseguran que en España es conveniente desarrollar un pensamiento propio, que integre críticamente las nuevas aportaciones metodológicas y desarrolle nuevos enfoques e instrumentos. La autora de esta recensión no puede dejar de alegrarse de esta invitación a la práctica del ensayo, por su dedicación personal a la literatura y a la filosofía, y aprovecha para aportar al público lector de Revisiones en Salud Pública y de esta recensión, la aclaración, por si fuera necesaria, de que un ensayo contiene la reflexión de su autor o autora acerca de un tema sobre el que previamente se ha documentado con rigor y exhaustividad, y que se escribe para elaborar una nueva aportación sobre el mismo, reconociéndose en ello la temporalidad de las soluciones hasta entonces señaladas, así como el perspectivismo de cualquier aspecto de la realidad. Requiere además una buena elaboración literaria.

No se trata, por supuesto, de convertir el ámbito de la Salud Pública en un Liceo Aristotélico, sino, efectivamente y, de acuerdo con los autores de la presentación de este libro que se está comentando, animar a los profesionales de la Salud Pública en España a adquirir un espíritu crítico y reflexivo, así como a aportar a los demás las conclusiones del mismo. Hay en la actualidad un tema que puede servir para ilustrar esta llamada de atención, además del que este mismo libro ofrece sobre la dicotomía entre las metodologías cuantitativas y cualitativas. Se trata del éxito que está teniendo en nuestro ámbito la llamada «Medicina basada en la evidencia», de la que no se ha podido leer ninguna reflexión crítica sobre la misma en nuestro país, a pesar de que sería necesaria y fructífera. Por otra parte afecta directamente a los criterios de aceptación de los trabajos en las revistas científicas, por lo que además sería muy conveniente la apertura de un debate en este sentido.

Entrando ya en el contenido de este quinto volumen de Revisiones en Salud Pública, se publican en él trabajos que se agrupan fun- 
damentalmente en dos secciones: la de trabajos Originales y la de los trabajos que se publican bajo el epígrafe «Debate». Además, se incluye la Declaración de Ljubliana sobre la Reforma de los Servicios Sanitarios, así como dos índices relacionados con los diferentes volúmenes de la serie, uno alfabético acumulado referido a los volúmenes 4 y 5 , y otro de autores que incluye a todos los de los cinco números de la publicación.

Entre los trabajos publicados en la sección de originales, merece especial atención por su interés Esperanza de vida saludable: pasado y presente de un indicador con futuro, que trata del cuestionamiento del indicador esperanza de vida en las circunstancias que actualmente caracterizan la mortalidad, por lo que se propone el indicador esperanza de vida saludable, se explican los diferentes métodos que existen para calcularlo y se hacen recomendaciones para su uso en España.

En el trabajo La organización y la práctica de la medicina general/de familia en Europa, se hace una revisión de las diferentes organizaciones de la medicina general (consulta en solitario, centros de salud, trabajo en grupo y policlínicos), forma de pago (por acto, por capitación y por salario) y su relación con diferentes variables de la práctica clínica: cargas de trabajo, derivación a especialistas, prescripción farmacéutica y resultados, medidos en términos de satisfacción de la población y mortalidad. Los autores concluyen que las asociaciones encontradas no son significativas y piensan que la información analizada es útil para diferentes tipos de profesionales.

El artículo original Qué es la investigación en servicios de salud de Vicente Navarro, denuncia de forma valiente el contenido de la Antología de cien artículos realizada por la OPS sobre investigación de servicios sanitarios, en la que los cien artículos elegidos, a pesar de tener una excelente y ya reconocida calidad, sólo contribuirán a re- producir el esquema existente, por no tener en cuenta para la solución necesaria de los problemas de salud en las Américas, las características macrosociales y macroeconómicas que influyen en ella. Por ello, el Sur volverá a salir perjudicado, tanto si se refiere al espacio geográfico, como si se refiere a los habitantes de la parte Norte de América que, sin embargo, siguen siendo el Sur. Sobre el mismo tema, el trabajo El reto de la promoción de la salud con equidad en el proceso de desarrollo de América Latina mantiene, al contrario que el anterior, un tono excesivamente conformista. En este trabajo se vuelve a plantear el viejo tema de las competencias del sistema sanitario. Más que asumir competencias en sectores ajenos a la salud, la solución pasaría porque todos los sectores tuvieran como principal objetivo de su actividad la salud de nuestro planeta y la de las poblaciones que lo habitan, por lo que una amplia parte de la política en general debería ser política de salud pública, lo que permitiría dejar a la Administración Sanitaria la suya propia, es decir la Política Sanitaria, que por supuesto sería parte importante de la anterior. Este trabajo resulta frío y anacrónico y su contenido entra en contradicción consigo mismo, ya que, aunque en su último epígrafe aboga por la cautela a la hora de importar modelos de intervención en América Latina, en sus 57 referencias bibliográficas sólo existen seis de trabajos realizados directamente en ese lugar, estando el resto de la información utilizada, elaborada en Europa y Amćrica del Norte.

En el trabajo Conexión informatizada de ficheros: su uso en los registros de cáncer de base poblacional» se expone en qué consiste la CIF, la cual se ocupa del desarrollo de la teoría probabilística y los sistemas de computación para que hagan el proceso de conexión de ficheros viable y válido. La teoría se desarrolló sobre todo en Canadá. Se comentan tanto el método probabilístico como el determinista, las ventajas de ambos en cada caso, la indicación de cada uno dependiendo de las características de la información a aparear, y los problemas éticos y 
legales que pueden surgir en su uso, y que se relacionan con la confidencialidad de los datos sobre las personas, por lo que resulta novedoso e interesante de leer.

\section{Inferencias, Philips Morris y factor de} impacto bibliográfico, introduce una crítica sobre la epidemiología y su capacidad para hacer «inferencia e inferencias», refiriéndose con el singular a la de apellido estadística, y con el plural a la del proceso de evaluación de la causalidad. Todo ello como fundamento para criticar la campaña publicitaria de Philips Morris durante la primavera de 1996, que pretendía demostrar la no tan grande perniciosidad del hábito de fumar. Es válida la lectura de este trabajo para conectar con el tema de la subjetividad del investigador en cualquier tipo de investigación. Es, sin embargo, desgraciada la referencia que el autor hace a la Sra Thacher, porque no es la persona más indicada para ser utilizada como modelo de defensa de libertades y porque el contenido de dicha referencia (No existe una cosa como la sociedad, únicamente existen familias e individuos) es lamentable.

En la sección de Debate se incluyen dos temas: el primero, manejado por los editores con más acierto que el segundo, se refiere a la necesaria revisión del modelo de gestión hospitalaria. Intervienen en él profesionales de la misma que saben lo que están diciendo y que tienen autoridad y autoría suficiente para decirlo. No sucede lo mismo con el segundo tema elegido por los editores para debatir: el que se propone sobre el uso de las metodologías cualitativas de investigación en salud pública. Aquí volvemos a conectar con la presentación de este quinto volumen de Revisiones en Salud Pública. Al tiempo que se invita a los lectores a la práctica del género del ensayo, se les anima al desarrollo en España de un pensamiento propio. Pues bien, en determinados temas este pensamiento propio ya existe pero, desgraciadamente, es despreciado en pro, entre otras causas, de poder citar el mayor número posible de referencias en inglés. Es el caso de la metodología cualitativa, ya que todos los trabajos incluidos en este volumen llevan la tristeza de que todas las citas bibliográficas son extranjeras. Hay que suponer además, que se desconoce el hecho de que fue en este país dónde hace más de treinta años nació el grupo de discusión, modalidad más característica de la metodología cualitativa, basándose en el reconocimiento de que el discurso social (el de esa sociedad que dice Benedetto Terracini que dice la Thacher que no existe) es susceptible de ser analizado e interpretado de cara a la investigación de múltiples y diferentes objetos de estudio. Independientemente de que fueran sociólogos o de otras profesiones no médicas, Jesús Ibañez, Alfonso Ortí, Fernando Conde y un largo etcétera de investigadores españoles, han realizado y publicado trabajos teóricos y prácticos de investigación con y sobre la metodología cualitativa en el campo de la salud, y sus bases teóricas son más profundas, fundamentadas y desarrolladas que cualquier libro o artículo que sobre el mismo tema se haya publicado en Estados Unidos, Reino Unido o Australia. El que esté interesado en ello, puede consultar «Más allá de la Sociología», «Del algoritmo al sujeto», ambos de Jesús Ibáñez; «Métodos y Técnicas cualitativas de investigación en ciencias sociales»... Lo que anima a escribir estas palabras es la intención de que conozca la existencia de estos textos cualquier persona que esté interesada en informarse y formarse en la metodología cualitativa. También en otras revistas del ámbito de las ciencias de la salud, es permanente encontrarse con este panorama e incluso peor, ya que, en lugar de aprender a hacer grupos de discusión, las personas que en nuestro país están aprendiendo metodología cualitativa, lo están haciendo con grupos focales, de origen norteamericano, los cuales producen un discurso mucho menos rico que el del grupo de discusión español y francés, porque en la metodología cualitativa no se trata de preguntar sino de escuchar, no se trata de que los sujetos investigados respondan, sino de que hablen, sin estar influidos por el enunciado de ninguna pregunta, que, para empezar, ya está informando a los par- 
ticipantes en el grupo de los intereses del investigador y condicionando, por ello, la respuesta. Por otra parte, en la metodología cualitativa se busca la producción de un discurso que, tras su análisis e interpretación, permitirá la elaboración de unos resultados que nunca necesitarán de la significación estadística para ser válidos, porque de hecho, ni la persiguen ni la pueden perseguir.

De acuerdo con la intervención de Fernando García Benavides, no se trata de que los epidemiólogos dejen de hacer su trabajo, pero tampoco simplemente de que los investigadores cualitativos hagan el suyo, sino que es necesario que, en general, epidemiólogos, gerentes de servicios sanitarios y sociales, planificadores, etc, conozcan los usos de esta metodología y sepan leer las investigaciones realizadas con ella, con el fin de que sus resultados y conclusiones sean utilizados por los pro- fesionales que se dedican a las ciencias de la salud, sean o no investigadores con metodología cualitativa.

Resumiendo y para terminar esta recensión, el quinto volumen de la colección Revisiones en Salud Pública contiene intervenciones que son interesantes, tanto en función del tema como de los autores que lo han tratado; entre ellas se incluye la propia presentación de los editores; otras intervenciones que simplemente no son interesantes, bien porque no lo era el tema, bien porque no han sido bien realizadas; y otras en las que a pesar de haberse elegido bien el tema, no se ha hecho, sin embargo, una buena elección de los autores de los trabajos. En conclusión, el libro no es perfecto, pero tampoco es desechable y desde aquí se anima a sus lectores a que hagan un ejercicio de crítica sobre el mismo, para comenzar a seguir los consejos de sus editores. 